\title{
Creative Al Through Evolutionary Computation: Principles and Examples
}

\author{
Risto Miikkulainen ${ }^{1}$ (D)
}

Received: 1 August 2020 / Accepted: 22 February 2021 / Published online: 23 March 2021

(c) The Author(s), under exclusive licence to Springer Nature Singapore Pte Ltd. part of Springer Nature 2021

\begin{abstract}
The main power of artificial intelligence is not in modeling what we already know, but in creating solutions that are new. Such solutions exist in extremely large, high-dimensional, and complex search spaces. Population-based search techniques, i.e. variants of evolutionary computation, are well suited to finding them. These techniques make it possible to find creative solutions to practical problems in the real world, making creative AI through evolutionary computation the likely "next deep learning."
\end{abstract}

Keywords Evolutionary computation $\cdot$ Machine creativity $\cdot$ Neuroevolution $\cdot$ Surrogate modeling $\cdot$ Decision making $\cdot$ Realworld applications

\section{Introduction}

In the last decade or so we have seen tremendous progress in Artificial Intelligence (AI). AI is now in the real world, powering applications that have a large practical impact. Most of it is based on modeling, i.e. machine learning of statistical models that make it possible to predict what the right decision might be in future situations. For example, we now have object recognition, speech recognition, game playing, language understanding, and machine translation systems that rival human performance, and in many cases exceed it $[9,10,28]$. In each of these cases, massive amounts of supervised data exist, specifying the right answer to each input case. With current computational capabilities, it is possible to train neural networks to take advantage of the data. Therefore, AI works great in tasks where we already know what needs to be done.

The next step for AI is machine creativity. Beyond modeling there is a large number of tasks where the correct,

This article is part of the topical collection "Evolution, the New AI Revolution" guest edited by Anikó Ekárt and Anna Isabel Esparcia-Alcázar.

Risto Miikkulainen

risto@cs.utexas.edu

1 The University of Texas at Austin and Cognizant Technology Solutions, San Francisco, USA or even good, solutions are not known, but need to be discovered. For instance designing engineering solutions that perform well at low costs, or web pages that serve the users well, or even growth recipes for agriculture in controlled greenhouses are all tasks where human expertise is scarce and good solutions difficult to come by [5, 12-14, 25]. Methods for machine creativity have existed for decades. I believe we are now in a similar situation as deep learning was a few years ago: with the million-fold increase in computational power, those methods can now be used to scale up to realworld tasks.

This paper first identifies challenges in creative tasks, suggests how evolutionary computation may be able to solve them, and reviews three practical examples of Creative AI through Evolutionary Computation.

\section{Challenges in Machine Creativity}

Evolutionary computation is in a unique position to take advantage of that power, and become the next deep learning. To see why, let us consider how humans tackle a creative task, such as engineering design. A typical process starts with an existing design, perhaps an earlier one that needs to be improved or extended, or a design for a related task. The designer then makes changes to this solution and evaluates them. S/he keeps those changes that work well and discards those that do not, and iterates. The process terminates when 
a desired level of performance is met, or when no better solutions can be found - at which point the process may be started again from a different initial solution. Such a process can be described as a hill-climbing process (Fig. 1a). With good initial insight it is possible to find good solutions, but much of the space remains unexplored and many good solutions may be missed.

Interestingly, current machine learning methods are also based on hill climbing. Neural networks and deep learning follow a gradient that is computed based on known examples of desired behavior [17, 30]. The gradient specifies how the neural network should be adjusted to make it perform slightly better, but it also does not have a global view of the landscape, i.e. where to start and which hill to climb. Similarly, reinforcement learning starts with an individual solution and then explores modifications around that solution, to estimate the gradient $[29,34]$. With large enough networks and datasets and computing power, these methods have achieved remarkable successes in recent years.

However, the search landscape in creative tasks is likely to be less amenable to hill climbing (Fig. 1b). There are three challenges: (1) the space is large, consisting of too many possible solutions to be explored fully, even with multiple restarts; (2) the space is high-dimensional, requiring that good values are found for many variables at once; and (3) the space is deceptive, consisting of multiple peaks and valleys, making it difficult to make progress through local search.

\section{Evolutionary Computation Solution}

Evolutionary computation, as a population-based search technique, is in a unique position to meet these challenges. First, it makes it possible to explore many areas of the search space at once. In effect, evolution performs multiple parallel searches, not a single hill climb. By itself such parallel search would result in only a linear improvement, however, the main advantage is that the searches interact: if there is a good partial solution found in one of the searches, the others can immediately take advantage of it as well. That is, evolution finds building blocks, or schemata, or stepping stones, that are then combined to form better comprehensive solutions $[6,11,21]$.

This approach can be highly effective, as shown e.g. in the benchmark problem of multiplexer design [16]. Multiplexers are easy to design algorithmically: the task is to output the bit (among $2^{n}$ choices) specified by an $n$-bit address. However, when formulated as a search problem in the space of logical operations this problem is challenging because the search space grows very quickly, i.e. as $2^{2^{n+2^{n}}}$. There is, however, structure in that space that evolution can discover and utilize effectively. It turns out that evolution can discover solutions in extremely large cases, including the 70-bit multiplexer (i.e. $n=6$ ) with a search space of at least $2^{2^{70}}$ states. It is hard to conceptualize a number that large, but to give an idea, imagine having the number printed using a $10 \mathrm{pt}$ font on a piece of paper. It would take light 95 years to traverse from the beginning to the end of that number.

Second, population-based search makes it possible to find solutions in extremely high-dimensional search spaces as well. Whereas it is very difficult to build a model with highorder interactions beyond pairs or triples, the population

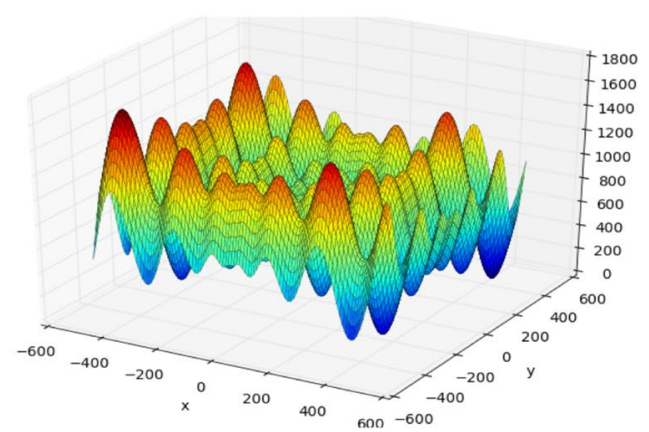

(b) Search Space in a Creative Domain
Fig. 1 Challenge of Creative Problem Solving. Human design process as well as deep learning and reinforcement learning can be seen as hill-climbing processes. They work well as long as the search space is relatively small, low-dimensional, and well behaved. However, creative problems where solutions are not known may require search in a large, high-dimensional space with many local optima. Populationbased search through evolutionary computation is well-suited for such problems: it discovers and utilizes partial solutions, searches along multiple objectives, and novelty. (Image credit: http://deap.readt hedocs.io/en/latest/api/benchmarks.html) 
represents such interactions implicitly, as the collection of actual combinations of values that exist in the good solutions in the population. Recombination of those solutions then makes it possible to collect good values for a large number of dimensions at once.

As an example, consider the problem of designing an optimal schedule for metal casting [4]. There are variables for number of each type of object to be made in each heat (i.e. melting process). The number of objects and heats can be grown from a few dozen, which can be solved with standard methods, to tens of thousands, resulting in billion variables. Yet, utilizing an initialization process and operators customized to exploit the structure in the problem, it is possible to find good combinations for them, i.e. find nearoptimal solutions in a billion-dimensional space. Given that most search and optimization methods are limited to several orders of magnitude fewer variables, this scaleup makes it possible to apply optimization to entire new category of problems.

Third, population-based search can be adapted naturally to problems that are highly deceptive. One approach is to utilize multiple objectives [3]: if search gets stuck in one dimension, it is possible to make progress among other dimensions, and thereby get around deception. Another approach is to emphasize novelty, or diversity, of solutions in search [33]. The search does not simply try to maximize fitness, but also favors solutions that are different from those that already exist. Novelty can be expressed as part of fitness, or a separate objective, or serve as a minimum criterion for selection, or as a criterion for mate selection and survival $[2,8,18,20,26]$.

For instance, in the composite novelty method [31], different objectives are defined for different aspects of performance, and combined so that they specify an area of search space with useful tradeoffs. Novelty is then used as the basis for selection and survival within this area. This method was illustrated in the problem of designing minimal sorting networks, which have to sort a set of $n$ numbers correctly, but also consist of as few comparator elements as possible (which swap two numbers), and as few layers as possible (where comparisons can be performed in parallel). The search space is highly deceptive because often the network structure needs to be changed substantially to make it smaller. Combining multiple objectives and novelty finds solutions faster and finds better solutions than traditional evolution, multiobjective evolution, and novelty search alone. The approach already found a new minimal network for 20 inputs [32].

Thus Evolutionary Computation has the right properties to solve challenging tasks that require creativity. The next three subsections review three examples on how this power can be put to use in discovering creative solutions in realworld applications.

\section{Designing Effective Web Interfaces}

The first example is Ascend by Evolv, an actual commercial application of evolutionary computation on conversion optimization, i.e. on designing web interfaces to make it more likely that a user will take the desired action on the page, such as signing them up, buying something, or requesting for more information [22, 24].

More specifically, the human expert defines a search space, consisting of a set of elements on the page, such as the heading text, size, and color, background image, and content order, possible values for each, and possible restrictions among their combinations. Page design can then be represented as a vector and optimized using genetic algorithms. Each candidate is evaluated by deploying it on the web. A sufficient number of actual users is directed to each candidate design, and how well they convert is measured.

Typically about 2000 users are needed to estimate a typical 1-4\% conversion rate for evolution to make progress. With a population of a few dozen candidates, Ascend usually discovers designs that are better than control in 10-20 generations. Ascend has been applied to hundreds of web interfaces across a variety of industries and search space sizes, and it routinely improves performance 10-200\% over the original human designs.

However, what is most interesting about Ascend is that it can discover creative solutions that human designers miss. The humans utilize principles of perceptual psychology and aesthetics, such as hierarchy, directionality, consistency, and clarity, but it turns out following them does not necessarily make the page effective. An example is shown in Fig. 2. While the control design is elegant, the design discovered by evolution is brash, using neon colors, contrast, and strong text. As a matter of fact, when evolution was running, it came up with similar designs so frequently that the designers labeled it "the ugly widget generator." However, this ugly widget performs $45 \%$ better than the control! This result suggests that there is still much that we do not know about factors that affect conversions, but evolution can nevertheless learn them and utilize them in creative design.

\section{Discovering Growth Recipes for Agriculture}

The second example takes advantage of surrogate modeling, a powerful extension that makes it possible to apply evolutionary creativity to many more problems in the real world. Whereas the Ascend designs could be evaluated in the real world with little cost, in many other domains, such 


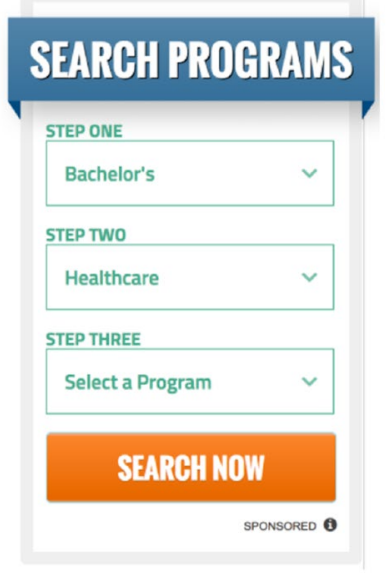

(a) Control

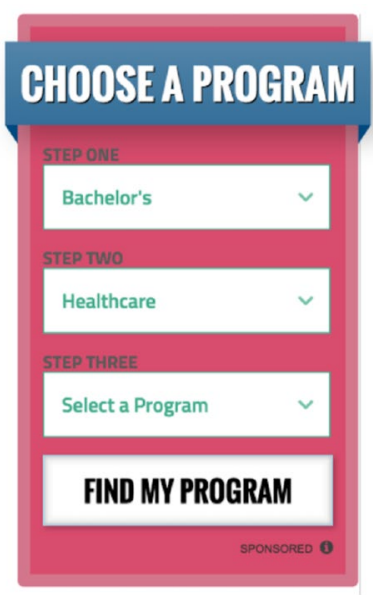

(b) Evolved
Fig. 2 A comparison of human design and evolutionary design for a sign-up widget in web design. a The original design is clear and consistent, according to general design principles. b The evolutionary design is brash and bold, and unlikely to be designed by humans. However, it converts $45 \%$ better, demonstrating that evolution can discover creative solutions that humans miss

as healthcare, finance, or mechanical design, such evaluations, especially of the most creative solutions, could be costly or dangerous.

A case in point is developing growth recipes for computer-controlled agriculture. That is, given vertical farming environments where the inputs such as water, temperature, nutrients, and light can be controlled at will, the challenge is to determine how those inputs should be set so that the plants grow as well as possible, for instance improving flavor, maximizing size, or minimizing cost. Whereas it takes a long time to grow a plant, a large number of recipes can be evaluated immediately with a surrogate model.

In a pioneering experiment, recipes were developed for optimizing flavor in basil, focusing on light variables such as wavelength, period, and UV component. Initially a few hundred recipes were implemented in real growth containers, representing known good recipes as well as recipes that covered the space more broadly. A surrogate model was trained on the resulting data, with flavor measured in terms of volatile composition of the plants. About a million recipes were then created through search and evaluated against the surrogate. In the end, the best ones were evaluated in real growth experiments.

In this process, a most remarkable discovery was made. Initially the maximum light period was set to $18 \mathrm{~h}$, assuming that the daily light cycle in the real world was a reasonable constraint. However, search quickly discovered that recipes at $18 \mathrm{~h}$ were the best. At that point, the daily cycle restriction was removed-and (as shown in Fig. 3), even better recipes were found with a light period of $24 \mathrm{~h}$ ! This result is

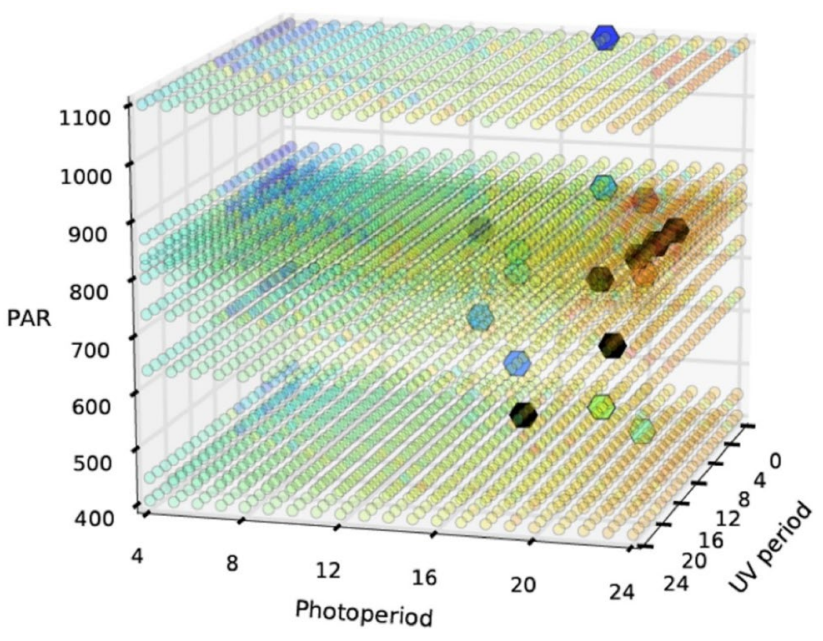

Fig. 3 Discovering a counterintuitive 24-h light period for computercontrolled agriculture. With the initial 18 -h restriction removed, evolution discovered that when the lights are always on, basil will develop more flavor. The axes represent the three light variables, with light period on the horizontal axis. The color of the small dots indicates their value predicted by the model ( red $>$ yellow $>$ green $>$ blue). The large dots are suggestions, and the darker dots are the most recent ones. In this manner, if given a search space free of human biases, evolution can discover effective, surprising solutions

counterintuitive and it was a surprise to the biologists in the team. It demonstrates how human biases can get in the way of discovering good solutions. Evolution does not have such biases, and if given enough freedom to explore, can create effective, surprising solutions.

\section{Finding Mitigation Strategies for COVID-19}

The third example is particularly topical at the time of this writing: Determining how various countries could implement non-pharmaceutical interventions (NPIs), such as school and workplace closings, restrictions on gatherings and events, and limitations on movement, to reduce the spread of the pandemic with minimal economic cost [23]. It is also significantly more complex in that, in addition to requiring a surrogate for evaluation, the solutions are strategies, represented by neural networks, instead of single points (such as web-page designs or growth recipes).

More specifically, the approach consists of first training a Predictor, i.e. a surrogate model, to predict how the number of cases would develop in the future, given a history of cases and NPIs in a country in the past, and an NPI strategy for the future. Using historical NPI data from the Oxford COVID19 government response tracker and case data from Johns Hopkins COVID-19 Data Repository, it was possible to train a recurrent LSTM neural network for this purpose. As opposed to traditional epidemiological models, such a model 
is purely phenomenological, includes all hidden interactions, and turned out to be surprisingly accurate, even given that the data is collected and used as the pandemic unfolds.

In the second step, Prescriptor neural networks, representing the NPI strategies, were then evolved, using the Predictor as a surrogate to evaluate how effective they were. Since there are two conflicting objectives (minimize cases vs. stringency of NPIs), the result is a Pareto front that trades off these objectives: Some prescriptors keep the number of cases down by locking down, others keep the society open with the cost of more cases. The decision maker can then select a desired tradeoff, and the Prescriptor will recommend the best NPI strategy that achieves it.

This process, Evolutionary Surrogate-assisted Prescription (ESP; [7]), made several creative discoveries. Early on it recognized that schools and workplaces are the most important NPIs; Indeed these are the two activities where people spend a lot of time with other people indoors, where it is possible to be exposed to significant amounts of the virus, as later became evident $[15,19,27]$. After the peak has passed and economies are opening up, it discovered that alternating between opening and closing schools and workplaces could be an effective way to lessen the impact on the economy while reducing cases (Fig. 4). While it may sound unwieldy, it has recently been suggested as a possibility [1]. Given the limited search space available for evolution, it is a creative solution for lifting the NPIs gradually. Coming out of a lockdown, it recognized that people are less likely to adhere to restrictions than they were going in, and therefore recommended more NPIs that can be enforced, such as restrictions on events and international travel. In this manner, the data-based modeling and evolutionary discovery was able to track the changing context of the pandemic, and recommend creative new responses. Counterfactual studies with past data suggested that they could have indeed been more effective than the actual NPIs implemented at the time [23]. For an interactive demo of the system, see https://evolu tion.ml/esp/npi.

\section{Conclusion}

To conclude, evolutionary computation is an AI technology that is on the verge of a breakthrough, as a way to take machine creativity to the real world. Like deep learning, it can take advantage of computational resources that are
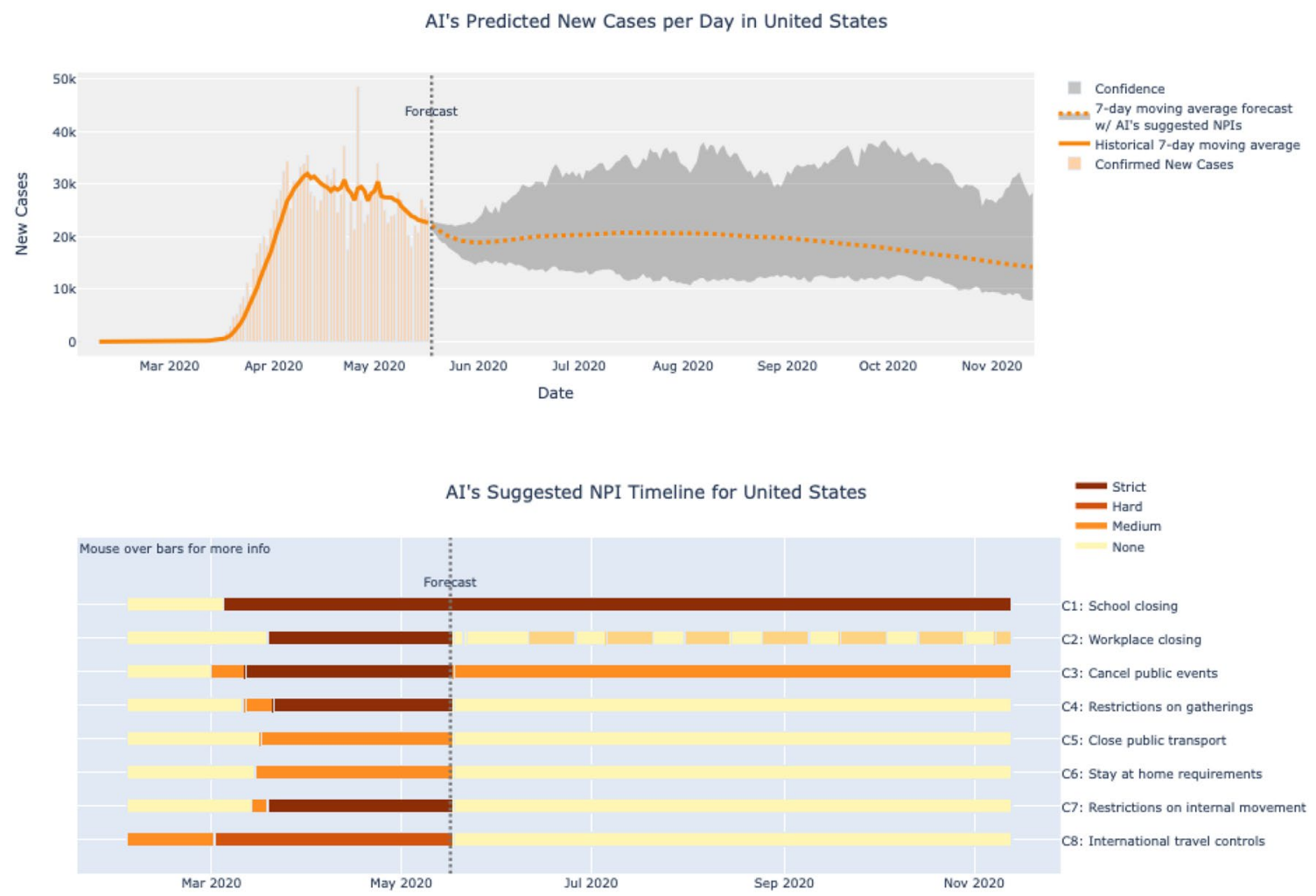

Fig. 4 An example creative solution for opening the economy after the COVID-19 peak had passed. The top plot shows the historical past and predicted future number of cases in the US on May 18th, 2020. The bottom plot illustrates the NPIs in effect or recommended during the same timeline, with color coding indicating their strin- gency. The system still recommends restrictions on schools, workplaces, and public events (top three rows), but suggests that opening and closing workplaces can be alternated, thus mitigating the effect on both economy and cases 
now becoming available. Because it is a population-based search method, it can scale with compute better than other machine learning approaches, which are largely based on hill climbing. With evolution, we should see many applications in the near future where human creativity is augmented by evolutionary search in discovering complex solutions, such as those in engineering, healthcare, agriculture, financial technology, biotechnology, and e-commerce, resulting in more complex and more powerful solutions than are currently possible.

Funding "Funding was provided in part by Defense Advanced Research Projects Agency (FA8750-18-C-0103), National Science Foundation (DBI-0939454).

\section{Declarations}

Conflict of interest The author declares that he has no conflict of interest.

\section{References}

1. Chowdhury R, Heng K, Shawon MSR, Goh G, Okonofua D, Ochoa-Rosales C, Gonzalez-Jaramillo V, Bhuiya A, Reidpath D, Prathapan S, Shahzad S, Althaus CL, Gonzalez-Jaramillo N, Franco OH, for COVID-19 Collaborative Group, T.G.D.I.S. Dynamic interventions to control COVID-19 pandemic: a multivariate prediction modelling study comparing 16 worldwide countries. Eur J Epidemiol. 2020. https://doi.org/10.1007/s1065 4-020-00649-w.

2. Cuccu G, Gomez F. When novelty is not enough. In: Proceedings of the 2011 international conference on applications of evolutionary computation, volume Part I. Berlin: Springer; 2011. p. 234-243.

3. Deb K, Agrawal S, Pratab A, Meyarivan T. A fast elitist non-dominated sorting genetic algorithm for multi-objective optimization: NSGA-II. In: Proceedings of parallel problem solving from nature (PPSN VI). Berlin: Springer VI; 2000. p. 849-858.

4. Deb K, Myburgh C. A population-based fast algorithm for a billion-dimensional resource allocation problem with integer variables breaking the billion-variable barrier in real-world. Eur J Oper Res. 2017;261:460-74.

5. Dupuis JF, Fan Z, Goodman E. Evolutionary design of discrete controllers for hybrid mechatronic systems. Int J Syst Sci. 2015;46:303-16.

6. Forrest S, Mitchell M. Relative building-block fitness and the building-block hypothesis. In: Whitley LD, editor. Foundations of genetic algorithms. New York: Elsevier; 1993. p. 109-26.

7. Francon O, Gonzalez S, Hodjat B, Meyerson E, Miikkulainen R, Qiu X, Shahrzad H. Effective reinforcement learning through evolutionary surrogate-assisted prescription. In: Proceedings of the genetic and evolutionary computation conference (GECCO-2020) New York: ACM; 2020. p. 814-82. http://nn.cs.utexas.edu/?franc on:gecco20

8. Gomes J, Mariano P, Christensen AL. Devising effective novelty search algorithms: a comprehensive empirical study. In: Proceedings of the 2015 annual conference on genetic and evolutionary computation. New York: ACM; 2015. p. 943-950.
9. Hassan Awadalla H, Aue A, Chen C, Chowdhary V, Clark J, Federmann C, Huang X, Junczys-Dowmunt M, Lewis W, Li M, Liu S, Liu TY, Luo R, Menezes A, Qin T, Seide F, Tan X, Tian F, Wu L, Wu S, Xia Y, Zhang D, Zhang Z, Zhou M. Achieving human parity on automatic Chinese to English news translation. Microsoft Research: Tech. rep; 2018.

10. Hessel M, Modayil J, van Hasselt H, Schaul T, Ostrovski G, Dabney W, Horgan D, Piot B, Azar MG, Silver D. Rainbow: combining improvements in deep reinforcement learning. 2017. arxiv :1710.02298

11. Holland JH. Adaptation in natural and artificial systems: an introductory analysis with applications to biology. Ann Arbor: Control and Artificial Intelligence, University of Michigan Press; 1975.

12. Hu J, Goodman ED, Li S, Rosenberg RC. Automated synthesis of mechanical vibration absorbers using genetic programming. Artif Intell Eng Des Manuf. 2008;22:207-17.

13. Ishida Lab: The N700 series Shinkansen (bullet train) (2018). https://www.sys.cs.tut.ac.jp/en/research-activities/research-intro duction/what-is-a-genetic-algorithm/2/. Accessed 29 Sept 2018.

14. Johnson AJ, Meyerson E, de la Parra J, Savas TL, Miikkulainen R, Harper CB. Flavor-cyber-agriculture: Optimization of plant metabolites in an open-source control environment through surrogate modeling. PLoS One. 2019. https://doi.org/10.1371/journ al.pone.0213918.

15. Kay J. COVID-19 superspreader events in 28 countries: Critical patterns and lessons. Quillette 2020. https://quillette. com/2020/04/23/covid-19-superspreader-events-in-28-countriescritical-patterns-and-lessons

16. Koza JR. A hierarchical approach to learning the Boolean multiplexer function. In: Rawlins GJE, editor. Foundations of genetic algorithms. Burlington: Morgan Kaufmann; 1991. p. 171-92.

17. LeCun Y, Bengio Y, Hinton G. Deep learning. Nature. 2015;521:436-44

18. Lehman J, Stanley KO. Revising the evolutionary computation abstraction: minimal criteria novelty search. In: Proceedings of the genetic and evolutionary computation conference. (GECCO2010). New York: ACM; 2010. p. 103-110

19. Lu J, Gu J, Li K, Xu C, Su W, Lai Z, et al. COVID-19 outbreak associated with air conditioning in restaurant, Guangzhou, China, 2020. Emerg Infect Dis. 2020. https://doi.org/10.3201/eid26 07.200764.

20. McQuesten P. Cultural enhancement of neuroevolution. Ph.D. thesis, Department of Computer Sciences, The University of Texas at Austin, Austin, TX. Technical Report AI-02-295; 2002. http:// nn.cs.utexas.edu/keyword?mcquesten:phd02.

21. Meyerson E, Miikkulainen R. Discovering evolutionary stepping stones through behavior domination. In: Proceedings of the genetic and evolutionary computation conference (GECCO 2017), New York: ACM; 2017. p. 139-146

22. Miikkulainen R, Brundage M, Epstein J, Foster T, Hodjat B, Iscoe N, Jiang J, Legrand D, Nazari S, Qiu X, Scharff M, Schoolland C, Severn R, Shagrin A. Ascend by Evolv: AI-based massively multivariate conversion rate optimization. AI Mag. 2020;42:44-60.

23. Miikkulainen R, Francon O, Meyerson E, Qiu X, Canzani E, Hodjat B. From prediction to prescription: evolutionary optimization of non-pharmaceutical interventions in the COVID-19 pandemic. arxiv:2005.13766 2020.

24. Miikkulainen R, Iscoe N, Shagrin A, Cordell R, Nazari S, Schoolland C, Brundage M, Epstein J, Dean R, Lamba G. Conversion rate optimization through evolutionary computation. In: Proceedings of the genetic and evolutionary computation conference (GECCO 2017). New York: ACM; 2017. pp. 1193-1199

25. Miikkulainen R, Iscoe N, Shagrin A, Rapp R, Nazari S, McGrath P, Schoolland C, Achkar E, Brundage M, Miller J, Epstein J, Lamba G. Sentient Ascend: AI-based massively multivariate conversion rate optimization. In: Proceedings of the thirtieth 
innovative applications of artificial intelligence conference. 2018. AAAI, Menlo Park, CA: AAAI Press. p. 7696-7703

26. Mouret JB, Doncieux S. Encouraging behavioral diversity in evolutionary robotics: an empirical study. Evol Comput. 2012;20:91-133.

27. Park SY, Kim YM, Yi S, Lee S, Na BJ, Kim C, et al. Coronavirus disease outbreak in call center, South Korea. Emerg Infect Dis. 2020. https://doi.org/10.3201/eid2608.201274.

28. Russakovsky O, Deng J, Su H, Krause J, Satheesh S, Ma S, Huang Z, Karpathy A, Khosla A, Bernstein MS, Berg AC, Li F. Imagenet large scale visual recognition challenge. 2014. arXiv:1409.0575

29. Salimans T, Ho J, Chen X, Sutskever I. Evolution strategies as a scalable alternative to reinforcement learning. 2017. arXiv :1703.03864

30. Schmidhuber J. Deep learning in neural networks: an overview. Neural Netw. 2015;61:85-117.

31. Shahrzad H, Fink D, Miikkulainen R. Enhanced optimization with composite objectives and novelty selection. In: Proceedings of the
2018 conference on artificial life. Cambridge, MA: MIT Press; 2018. p. 616-622. http://nn.cs.utexas.edu/?shahrzad:alife18

32. Shahrzad H, Hodjat B, Dolle C, Denissov A, Lau S, Goodhew D, Dyer J, Miikkulainen R. Enhanced optimization with composite objectives and novelty pulsation. In: Banzhaf W, Goodman E, Sheneman L, Trujillo L, Worzel B (eds) Genetic programming theory and practice XVII. New York: Springer; 2020. p. 275-293

33. Stanley KO, Lehman J. Why greatness cannot be planned: the myth of the objective. Berlin: Springer; 2015.

34. Zhang X, Clune J, Stanley KO. On the relationship between the OpenAI evolution strategy and stochastic gradient descent. 2017. arXiv:1712.06564.

Publisher's Note Springer Nature remains neutral with regard to jurisdictional claims in published maps and institutional affiliations. 